\title{
A Novel Approach for Video Frame Interpolation using Cubic Motion Compensation Technique
}

\author{
Ragina C. Ghutke, Ch. Naveen and Vishal R. Satpute \\ M. Tech. Scholar, Research Scholar, Assistant Professor, \\ VNIT Nagpur, India
}

\begin{abstract}
Increasing frame rates by frame interpolation is one of the main challenges in video processing. Motion estimation and motion compensation are two main keys of video frame interpolation algorithm. Motion estimation algorithms are used to get refine motion vectors while motion compensation algorithm assigns motion vectors accurately. Recent studies on video frame interpolation mainly focused on motion estimation algorithms, but along with that assigning motion vectors accurately is also an important issue. This paper concentrates on motion compensation. In this paper we have proposed a novel method for video frame interpolation using Cubic Motion Compensation technique which assigns motion vectors accurately. Instead of using two consecutive frames for motion estimation as in Conventional method, this paper considers four consecutive frames and calculates three different motion vectors. It is observed that proposed algorithm have relatively outperforms over conventional Motion compensated frame interpolation algorithm. For evaluation, average Peak Signal to Noise Ratio (PSNR) and average Structural Similarity (SSIM) are considered to compare the results. According to simulation results, using test sequences of different frame sizes, it can be observed that the proposed algorithm have effectively improves average PSNR of reconstructed frame by around $3 \mathrm{~dB}$ and average SSIM have increased upto $5 \%$ with reference to the traditional methods.
\end{abstract}

Keywords: Motion Estimation (ME), Motion Compensated Frame Interpolation (MCFI), Structural Similarity (SSIM), Video Graphic Array (VGA), Common Intermediate Format (CIF).

\section{INTRODUCTION}

Temporal frame interpolation upconverts frame rate by inserting new frames between the original frames of video sequences. This process plays significant role in modern video processing applications like frame rate up-conversion, low bit rate video coding, scalable video coding, slow motion playback, distributed video coding etc. To satisfy bandwidth requirement over internet or wireless networks, it is observed that, many a times frame dropping or filtering techniques are used. With emergence of new multimedia and television systems, it is required to convert frame rate between two different display formats with different frame rates for different display devices [2].

Temporal frame interpolation or Frame rate up conversion algorithms can be divided into two different methods. Simplest method includes repetition of frames or average of two frames and another method is motion compensated frame interpolation. Repetition of frames cause motion judder, while by averaging two frames to reconstruct the new frame leads to motion blur commonly referred as ghost artifacts.

Many motion compensated frame interpolation techniques are widely used for better quality [1-13]. Motion compensated frame interpolation algorithm have two main aspects i.e. motion estimation (ME) and motion compensation (MC). To achieve better performance in temporal frame interpolation, first it requires proper motion estimation to get accurate motion vectors and second priority is to accurately assign motion vectors in new interpolated frame. In this paper we are emphasizing on second issue of accurately assigning motion vectors.

Many efficient motion estimation algorithms have been proposed till date [10-13]. Previous studies adopt motion compensation technique discussed in section III-A. This paper, proposes a new motion compensation frame interpolation algorithm based on cubic interpolation technique. This approach improves quality of reconstructed frame.

The paper is organized as follows: First, the basic algorithm and existing work is described, then proposed cubic motion compensated algorithm is explained followed by performance evaluation of the proposed algorithm compared with existing motion compensated algorithm and conclusion of the work.

\section{THEORETICAL BACKGROUND}

Kang and Kim, [1] proposed unidirectional and bidirectional matching blocks in previous and current frames to achieve better visual quality. Wang et al, [2] proposed a technique in which moving objects are detected and segmented into blocks to perform motion compensated frame interpolation and also investigates the quality and complexity trade-off. Choi et al, [3] proposed a motion compensated frame interpolation method using bi-directional motion estimation. By segmentation, frame is divided into stationary background, moving object, covered and uncovered regions. Guo et al, [13] handles occluded region while frame rate up-conversion.

Motion estimation and motion compensation are two important methods in temporal frame interpolation. In this section idea of motion estimation (ME) algorithm is described. Motion estimation is done to reduce temporal redundancy i.e. to evaluate change between original adjacent frames. Motion Estimation (ME) method discussed in this paper is based on block matching algorithm. Process of getting best match block by block is called block based Motion Estimation. Forward motion estimation, backward motion estimation and 
bidirectional motion estimation are three different methods of ME. For getting best match in ME, there are some matching criteria [16-17],

- Mean Squared Error (MSE)

- Mean Absolute Difference (MAD)

- Sum of Absolute Difference (SAD)

- Sum of Absolute Transformed Difference (SATD)

- Sum of Bidirectional Absolute Difference (SBAD)

In this paper we are using MAD as matching criteria, after experimental results compared with MSE. There are different search algorithms of Motion Estimation (ME) [17],

- Full Search algorithm (Exhaustive search)

- 2D Logarithmic Search Algorithm

- Cross Search

- $\quad$ Three Step Search (TSS) Algorithm

- One at a time Search Algorithm

- Diamond Search Algorithm

- Hierarchical motion estimation (HME)

- 3D-Recursive Search algorithm (3DRS) [11]

- Hierarchical 3DRS motion estimation [11]

In this paper, TSS algorithm is applied as it is simple and takes less computational time with achieving approximate estimation [16].

In block matching motion estimation, two consecutive frames are considered. Here we are considering forward motion estimation wherein, current frame is divided into nonoverlapping blocks of size $\mathrm{N}^{*} \mathrm{~N}$ and each block is searched in next frame within a search window of size $(\mathrm{N}+2 \mathrm{P})$ [17]. $\mathrm{P}$ is a search window range. Assuming $f_{k-1}$ and $f_{k+1}$ as current frame and next frame respectively, motion vectors $V\left(M V_{x}, M V_{y}\right)$ are estimated using MAD as given in Eq. 1 and Eq. 2 [17]. $M V_{x}$, $M V_{y}$ are motion vectors.

$$
\begin{aligned}
& M A D(i, j)=\left(\frac{1}{N^{*} N}\right) *\left[\sum_{x=0}^{N-1} \sum_{y=0}^{N-1} \mid f_{k-1}(x, y)-f_{k+1}(x+i, y+j)\right] \\
& V\left(M V_{x}, M V_{y}\right)=\arg \min \{M A D(i, j)\}
\end{aligned}
$$

Where, $i$ and $j$ represents pixel positions of frames. After finding motion vectors, constructing the interpolated frame using motion compensation is very essential part.

Here we introduce the concept of applying cubic interpolation for motion compensation to get better interpolated frame.

\section{PROPOSED WORK}

Conventional motion compensation algorithm is discussed in section-A, followed by proposed cubic motion compensated frame interpolation algorithm in section-B.

\section{Conventional Motion Compensation frame interpolation Algorithm}

Using motion vectors from Eq. 2, the conventional motion compensation algorithm can be applied as,

$$
f_{k}(x, y)=\frac{1}{2}\left\{f_{k-1}(x, y)+\left[f_{k+1}\left(x-\frac{M V x}{2}, y-\frac{M V y}{2}\right)\right]\right\}
$$

$f_{k}(x, y)$ is an intermediate frame to be interpolated. For comparing proposed algorithm with conventional algorithm,
Eq. 3 [4] is used for motion compensated frame interpolation. Eq. 3 is linear motion compensated interpolation as it is averaging two frames using motion vectors.

It is proven that for spatial interpolation, bicubic interpolation method outperforms over bilinear interpolation method. So this paper uses the cubic motion compensated frame interpolation algorithm to improve the reconstructed quality of intermediate frame.

\section{Cubic Motion Compensated Frame Interpolation Algorithm}

Instead of using conventional linear Motion Compensated Frame Interpolation (MCFI), cubic interpolation is introduced in this paper. Instead of two successive frames for frame interpolation, four successive frames are considered in the cubic interpolation technique. Say $f_{k}$ is an intermediate frame to be interpolated, $f_{k-1}$ is a current frame, $f_{k-2}$ is a previous frame, $f_{k+1}$ is a next frame and $f_{k+2}$ is a next to next frame. The equation for calculating intermediate frame $f_{k}$ is given by Eq. 4, $f_{k}(x, y)=\left[B\left(t_{1}\right) * Z_{1}\right]+\left[B\left(t_{2}\right) * Z_{2}\right]+\left[B\left(t_{3}\right) * Z_{3}\right]+\left[B\left(t_{4}\right) * Z_{4}\right]$

Where,

$$
\begin{aligned}
& Z_{1}=f_{k-2}\left(x-\frac{M V x_{2}}{2}, y-\frac{M V y_{2}}{2}\right) \\
& Z_{2}=f_{k-1}(x, y) \\
& Z_{3}=f_{k+1}\left(x-\frac{M V x_{1}}{2}, y-\frac{M V y_{1}}{2}\right) \\
& Z_{4}=f_{k+23}\left(x-\frac{M V x_{3}}{2}, y-\frac{M V y_{3}}{2}\right)
\end{aligned}
$$

Where, $x$ and $y$ represents pixel positions of frames and $M V_{x 1}$, $M V_{y 1}, M V_{x 2}, M V_{y 2}, M V_{x 3}$ and $M V_{y 3}$ represents motion vectors. $V\left(M V_{x l}, M V_{y l}\right)$ is estimated motion vector between frames $f_{k-1}$ and $f_{k+1}$ by forward ME, $V\left(M V_{x 2}, M V_{y 2}\right)$ is estimated motion vectors between frames $f_{k-1}$ and $f_{k-2}$ by forward $\mathrm{ME}$, $V\left(M V_{x 3}, M V_{y 3}\right)$ is estimated motion vectors between frames $f_{k-1}$ and $f_{k+2}$ by backward $\mathrm{ME}, B\left(t_{i}\right)$ (where, $\left.i=1,2,3,4\right)$ is an interpolation kernel which is known as B-spline function, $t_{i}$ are data positions.

Equations for interpolation are given from Eq. 6 to Eq. 12 [14]. Let, $B\left(t_{i}\right)=u\left(s_{i}\right)$, where $u\left(s_{i}\right)$ is calculated by Eq. 6-Eq. 12. If $f$ is original function and $\mathrm{g}$ is its corresponding interpolation function, then $g\left(x_{k}\right)=f\left(x_{k}\right)$ for $x_{k}$ as interpolation node. The interpolation function can be written as Eq. 6,

$$
g(x)=\sum_{k}\left(c_{k} u\left(\frac{x-x_{k}}{h}\right)\right)
$$

Where, $g(x)$ is interpolation function. $C_{k}$ is control point which is a parameter depend on known data point or $k^{\text {th }}$ sample. It can be also written as $C_{k}=f\left(x_{k}\right) . u$ is the interpolation kernel. $h$ is sampling increment. $h=x_{i+1}-x_{i} . x_{k}$ is interpolation node. $x$ is a point where data is to be interpolated and it is between $x_{i+1}$ and $x_{i}$ which are consecutive interpolation nodes. Say, $x$ is a point in an interval $\left[x_{0}, x_{1}\right]$. For this $x, k=-1,0,1,2$. The difference between two interpolation nodes $x_{i}$ and $x_{k}$ is given by Eq. 7 ,

$x_{i}-x_{k}=(i-k) * h$

Let, 
$s=\frac{x-x_{i}}{h}$

$\frac{x-x_{i}}{h}=\frac{x-x_{i}+x_{i}-x_{k}}{h}=s+i-k$

Eq. 6 can be written as Eq. 10,

$g(x)=\sum_{k}\left(c_{k} u(s+i-k)\right)$

For $i=k$, Eq. 10 becomes Eq. 11,

$$
g(x)=\sum_{k}\left(c_{k} u(s)\right)
$$

The cubic interpolation kernel is given by Eq. 12,

$$
\left.\begin{array}{ll}
u(s)=\frac{3}{2}|s|^{3}-\frac{5}{2}|s|^{2}+1 & 0<|s|<1 \\
u(s)=-\frac{1}{2}|s|^{3}+\frac{5}{2}|s|^{2}-4|s|+2 & 0<|s|<1 \\
u(s)=0 & 2<|s|
\end{array}\right\}
$$

Outline of proposed work is shown as a block diagram in Figure 1. Flow chart summarizes all steps in entire process of temporal frame interpolation.

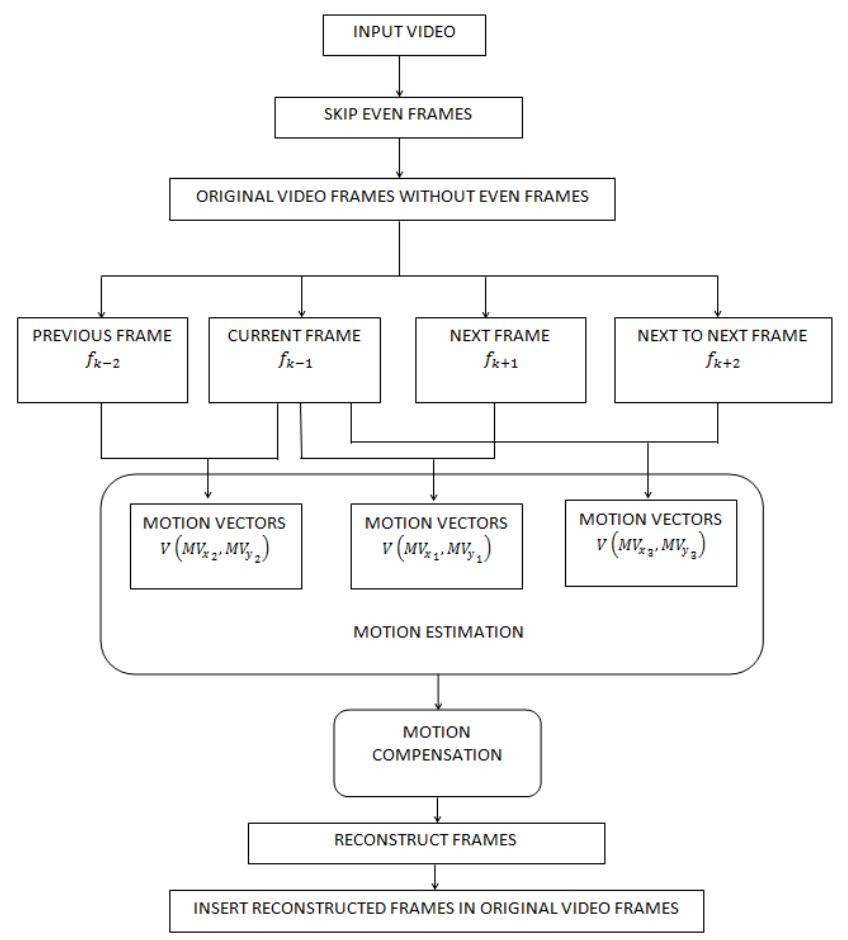

Figure 1: Flow Chart of Proposed Algorithm

\section{PERFORMANCE EVALUATION}

All simulations has been done using MATLAB and tests were executed on an Intel(R) Core(TM) i7-4770 CPU@3.40GHz clock frequency and 32 GB of RAM. For simulation, video sequences of different frame sizes are taken. These videos are taken from different video databases [21-24]. To compare the cubic motion compensated interpolation method with the conventional motion compensated interpolation, a common motion estimation method i.e. TSS has been applied. Taking this motion estimation method as a common platform we have compared results obtained. For performance evaluation Peak Signal to Noise Ratio (PSNR), Mean Squared Error (MSE) and Structural Similarity (SSIM) have been used.

The proposed method has been applied to different video sequences with frame size varying from CIF (Common Intermediate Format) $352 * 288$ to HD1080p (High Definition) $1920 * 1080$ progressive. To verify proposed method, we have dropped all even frames intentionally from original sequence and reconstructed all dropped frames to compare them with the originally dropped frames. The reconstructed frames are mathematically compared with the original frames using the parameters PSNR, MSE and SSIM. Mean Squared Error is given by Eq. 13 [1, 19],

$$
M S E=\frac{1}{M^{*} N} \sum_{x=0}^{M-1} \sum_{y=0}^{N-1}\left[f_{k}(x, y)-f_{k}^{\prime}(x, y)\right]^{2}
$$

Where $f_{k}$ is original frame and $f_{k}$, is reconstructed frame and $\mathrm{M}^{*} \mathrm{~N}$ is size of frame. Peak Signal to Noise Ratio is given by Eq. $14[1,19]$,

$$
P S N R=10 *\left[\log _{10}\left(\frac{255 * 255}{M S E}\right)\right]
$$

Where, MSE is mean Square error. Structural Similarity index (SSIM) is given by Eq. 15 \& Eq. 16 [1, 15, 20].

$$
\begin{aligned}
& \operatorname{SSIM}(x, y)=L(x, y) * C(x, y) * S(x, y)=\frac{4 \mu_{x} \mu_{y} \sigma_{x y}}{\left(\mu_{x}^{2}+\mu_{y}^{2}\right)\left(\sigma_{x}^{2}+\sigma_{y}^{2}\right)} \\
& L(x, y)=\frac{2 \mu_{x} \mu_{y}}{\left(\mu_{x}^{2}+\mu_{y}^{2}\right)}, C(x, y)=\frac{2 \sigma_{x} \sigma_{y}}{\left(\sigma_{x}^{2}+\sigma_{y}^{2}\right)}, S(x, y)=\frac{\sigma_{x y}}{\left(\sigma_{x}+\sigma_{y}\right)}
\end{aligned}
$$

$L(x, y)$ is luminance comparison, $C(x, y)$ is contrast comparison and $S(x, y)$ is structure comparison. These are three similarity measurement comparisons. Consider $I_{x}$ and $I_{y}$ as original and reconstructed frame respectively. $u_{x}, u_{y}, \sigma_{x}, \sigma_{y}$ and $\sigma_{x y}$ denote the mean, the variance and the covariance of $I_{x}$ and $I_{y}$ respectively.

For evaluation, initially motion vectors are calculated using TSS method. Table I shows video database used for evaluation of the proposed algorithm. In this work only gray scale videos are considered.

TABLE I. Video Parameters

\begin{tabular}{|c|c|c|c|c|}
\hline $\begin{array}{c}\text { Type of Video } \\
\text { (Resolution) }\end{array}$ & Video Name & $\begin{array}{c}\text { No. of } \\
\text { frames }\end{array}$ & $\begin{array}{c}\text { Frame } \\
\text { Rates }\end{array}$ & Source \\
\hline CIF (352*288) & Foreman.yuv & 300 & 30 & $\begin{array}{c}\text { VIPS } \\
{[21]}\end{array}$ \\
\hline VGA (640*480) & visor_1.avi & 65 & 16 & $\begin{array}{c}\text { Visor } \\
{[22]}\end{array}$ \\
\hline $\begin{array}{c}\text { HD 720p } \\
(1280 * 720)\end{array}$ & agh_src6_hrc0.avi & 300 & 25 & $\begin{array}{c}\text { VQ } \\
{[23]}\end{array}$ \\
\hline $\begin{array}{c}\text { HD 1080p } \\
(1920 * 1080)\end{array}$ & Boat.avi & 200 & 29 & {$[24]$} \\
\hline
\end{tabular}


TABLE II. Average PSNR \& Average SSIM In Tabular Form

\begin{tabular}{|c|c|c|c|c|}
\hline $\begin{array}{c}\text { Type Of } \\
\text { Video }\end{array}$ & \multicolumn{2}{|c|}{ Average PSNR (dB) } & \multicolumn{2}{c|}{ Average SSIM } \\
\cline { 2 - 5 } & $\begin{array}{c}\text { Conventional } \\
\text { MCFI }\end{array}$ & $\begin{array}{c}\text { Cubic } \\
\text { MCFI }\end{array}$ & $\begin{array}{c}\text { Conventional } \\
\text { MCFI }\end{array}$ & $\begin{array}{c}\text { Cubic } \\
\text { MCFI }\end{array}$ \\
\hline CIF & 24.2743 & 26.9561 & 0.7226 & 0.7921 \\
\hline VGA & 33.2069 & 36.0185 & 0.9729 & 0.9825 \\
\hline HD720p & 26.4010 & 29.3940 & 0.9429 & 0.9657 \\
\hline HD1080p & 27.3397 & 29.2548 & 0.8951 & 0.9391 \\
\hline
\end{tabular}

Experimental results are shown in Table II. Comparison between two approaches represented graphically in terms of average PSNR and average SSIM in Figure 2-Figure 3.

Figure 4-Figure 7 shows graphical representation of PSNR of each skipped frames in a video using conventional MCFI algorithm and cubic MCFI algorithm. Original frame and reconstructed frames of videos using conventional MCFI algorithm and cubic MCFI algorithm are shown in Figure 8Figure 11.

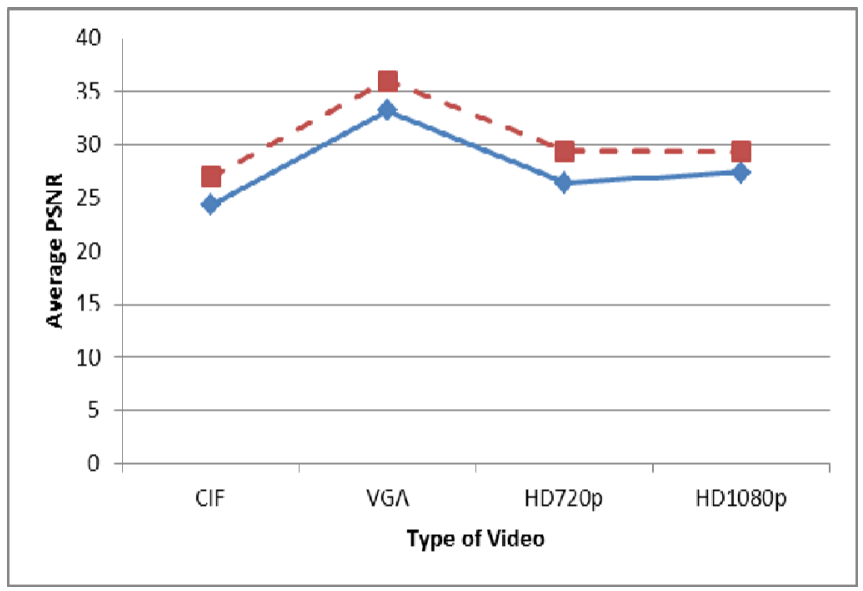

Figure 2: Graphical representation of average PSNR

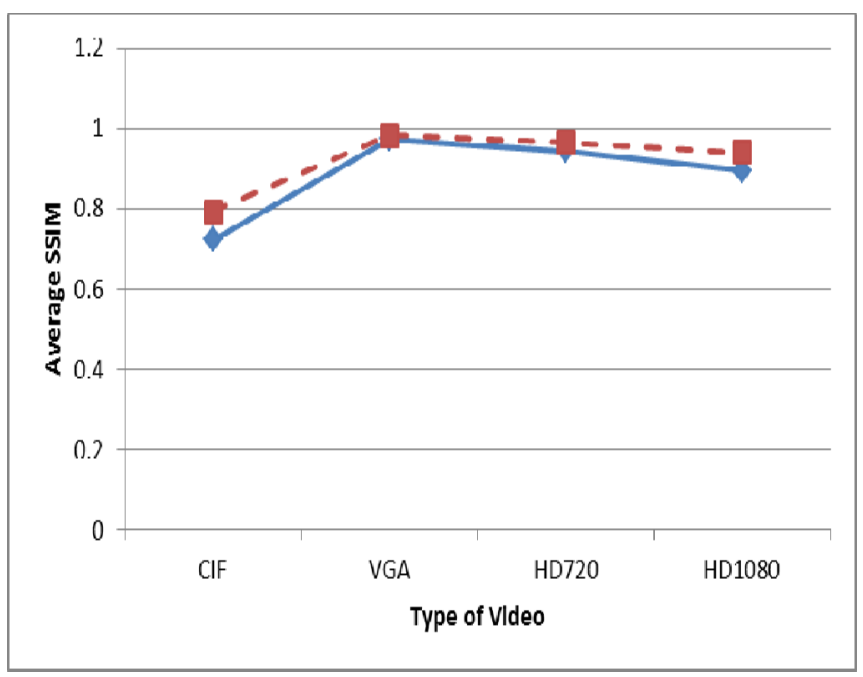

Figure 3: Graphical representation of average SSIM

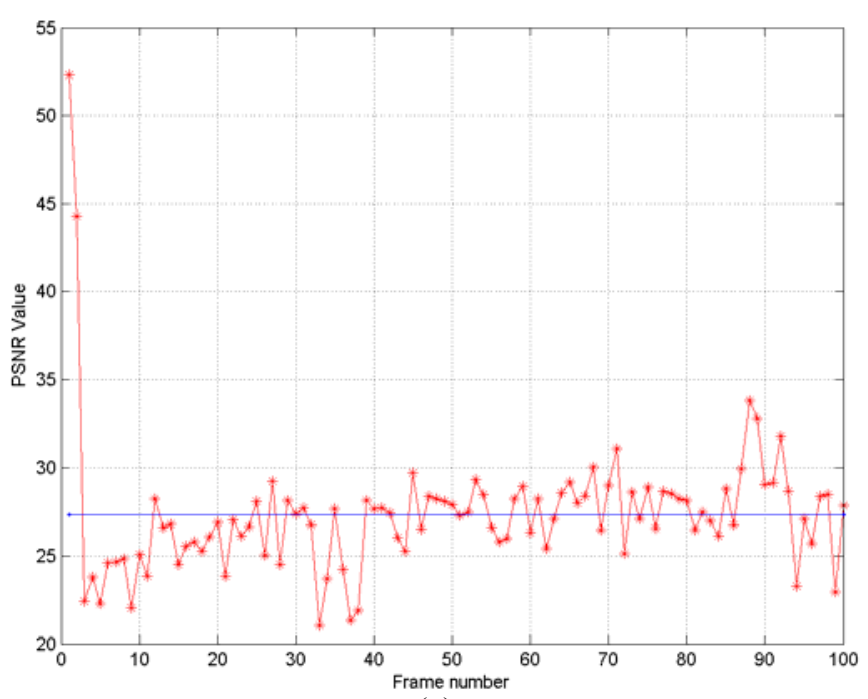

(a)

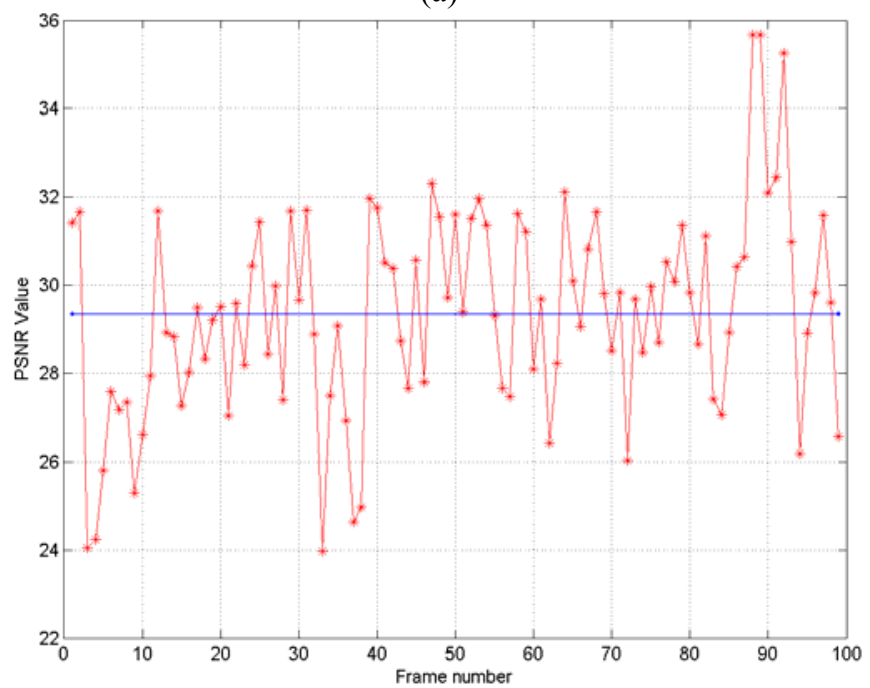

(b)

Figure 4: For HD1080p resolution video, graphical representation of PSNR of each skipped frames using; (a) conventional MCFI algorithm, (b) cubic MCFI algorithm.

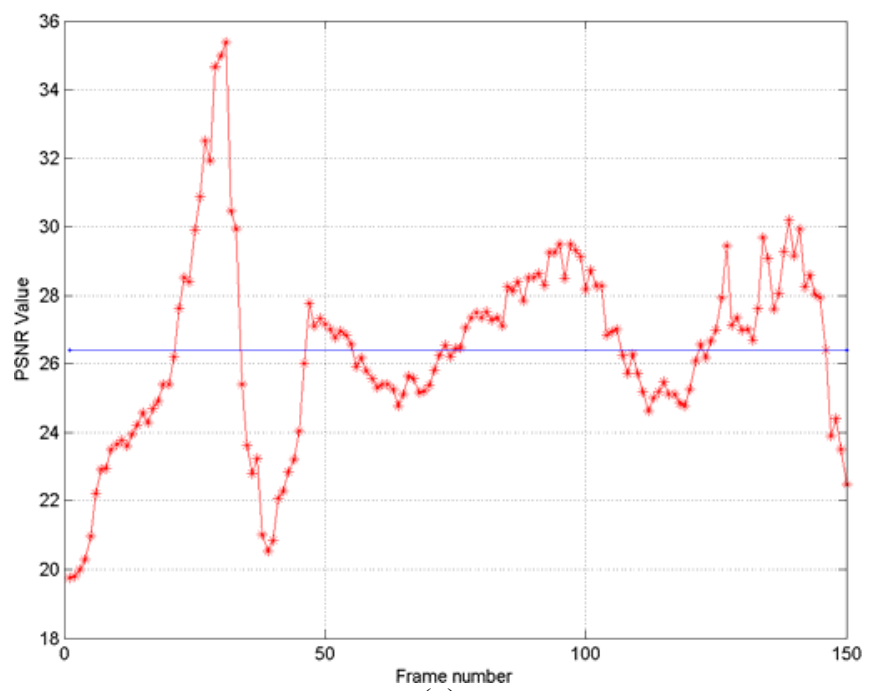

(a) 
International Journal of Applied Engineering Research ISSN 0973-4562 Volume 11, Number 10 (2016) pp. $7139-7146$

(C) Research India Publications. https://dx.doi.org/10.37622/IJAER/11.10.2016.7139-7146

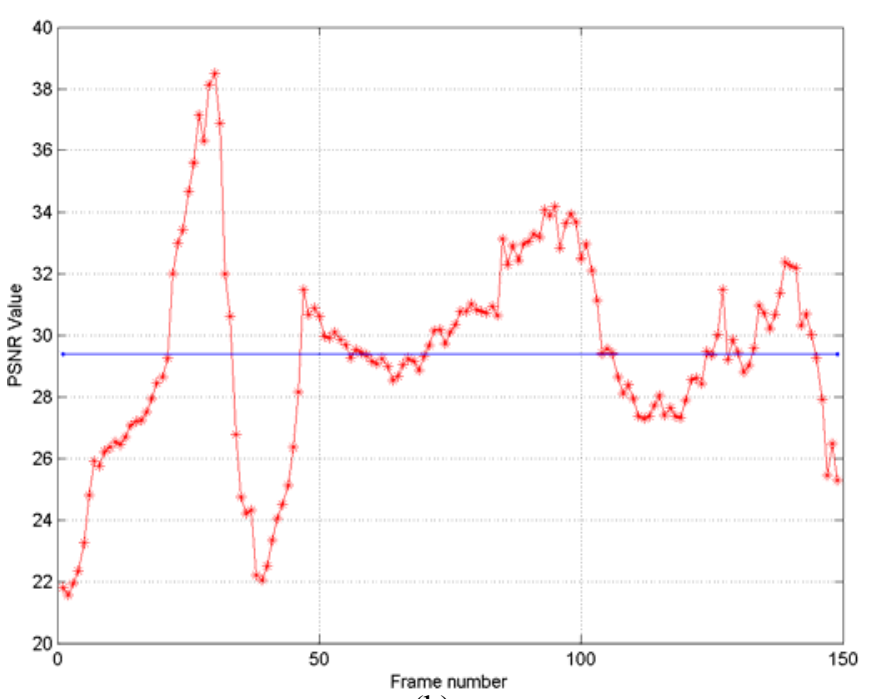

(b)

Figure 5: For HD720p resolution video, graphical representation of PSNR of each skipped frames using; (a) conventional MCFI algorithm, (b) cubic MCFI algorithm.

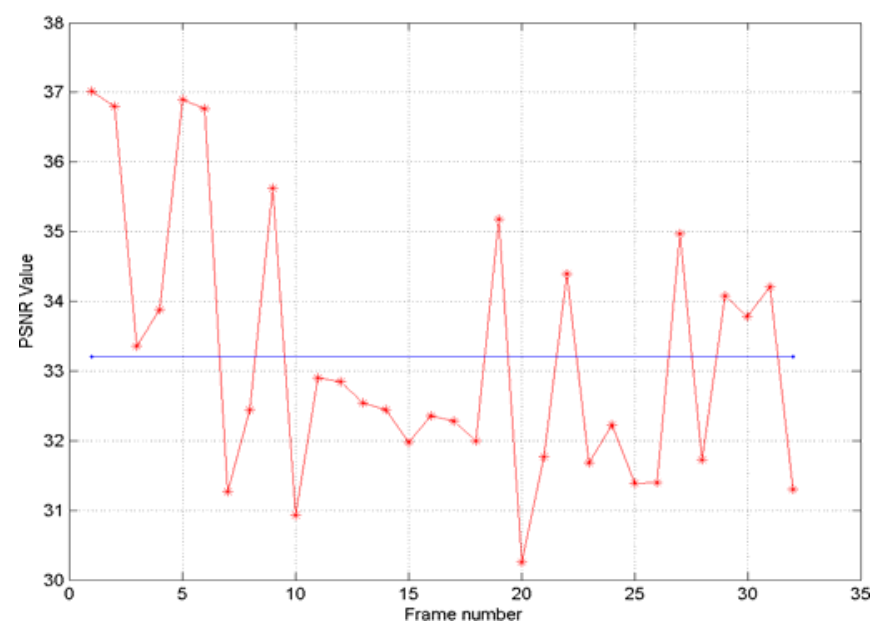

(a)

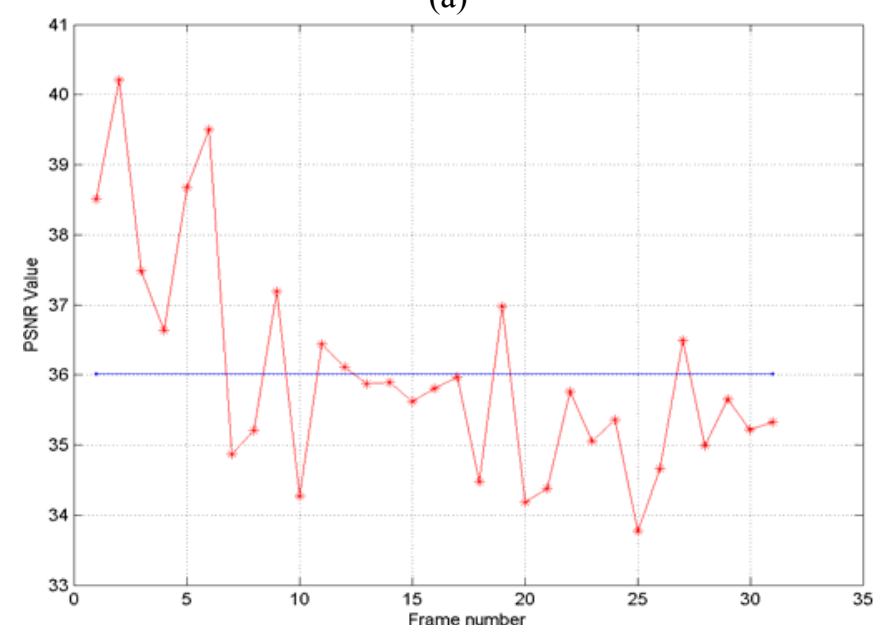

(b)

Figure 6: For VGA $640 * 480$ resolution video, graphical representation of PSNR of each skipped frames using; (a) conventional MCFI algorithm, (b) cubic MCFI algorithm.

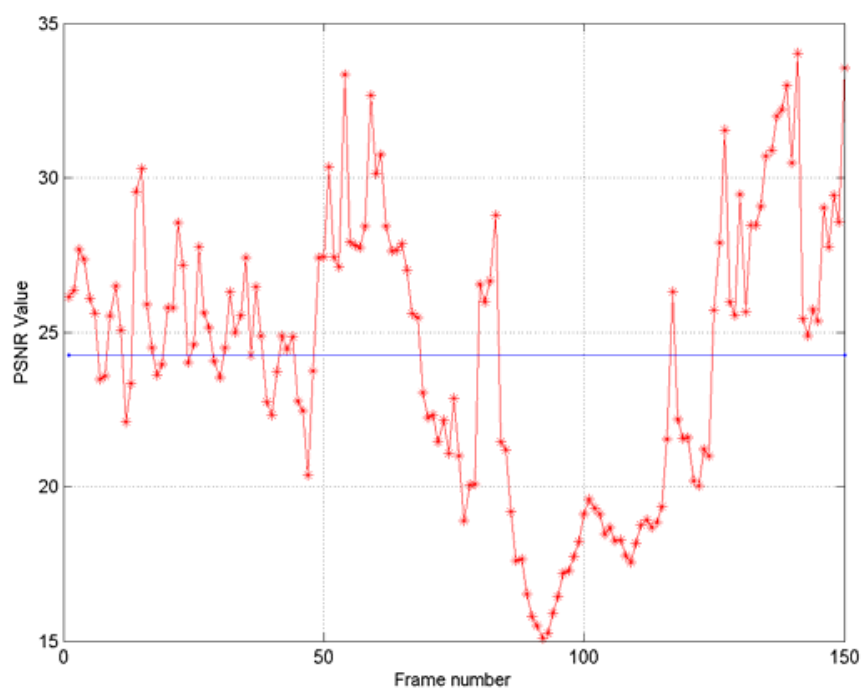

(a)

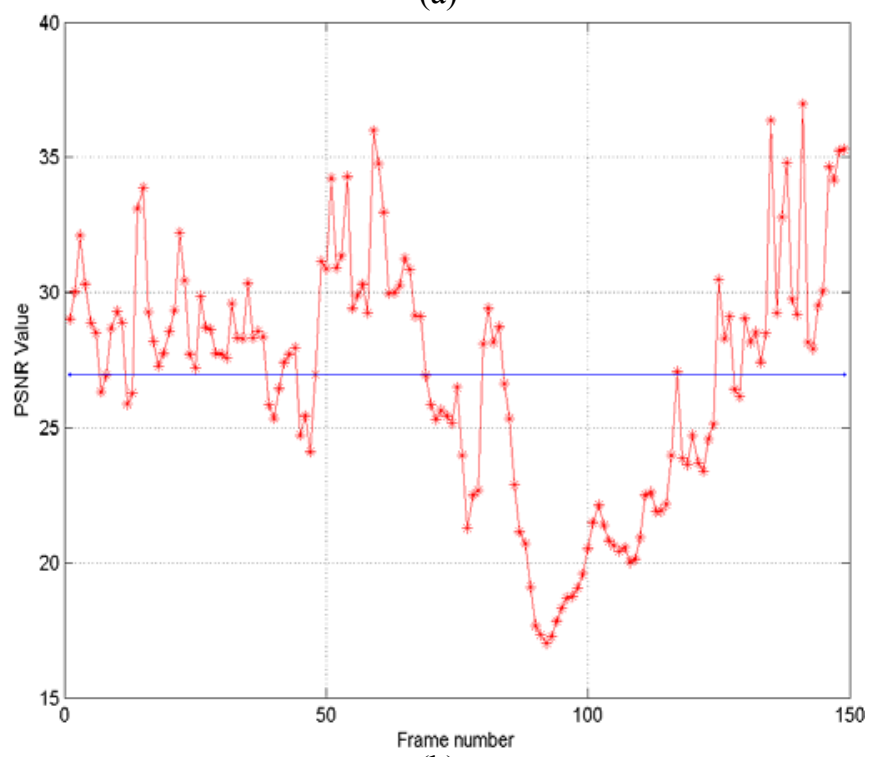

(b)

Figure 7: For CIF $352 * 288$ resolution video, graphical representation of PSNR of each skipped frames using; (a) conventional MCFI algorithm, (b) cubic MCFI algorithm.

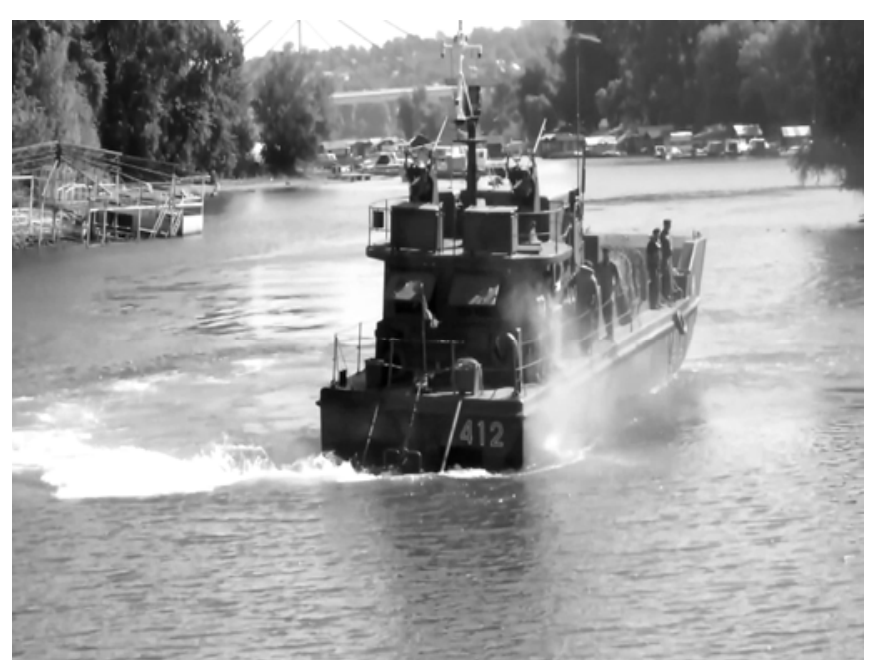

(a) 
International Journal of Applied Engineering Research ISSN 0973-4562 Volume 11, Number 10 (2016) pp. 7139-7146

(C) Research India Publications. https://dx.doi.org/10.37622/IJAER/11.10.2016.7139-7146

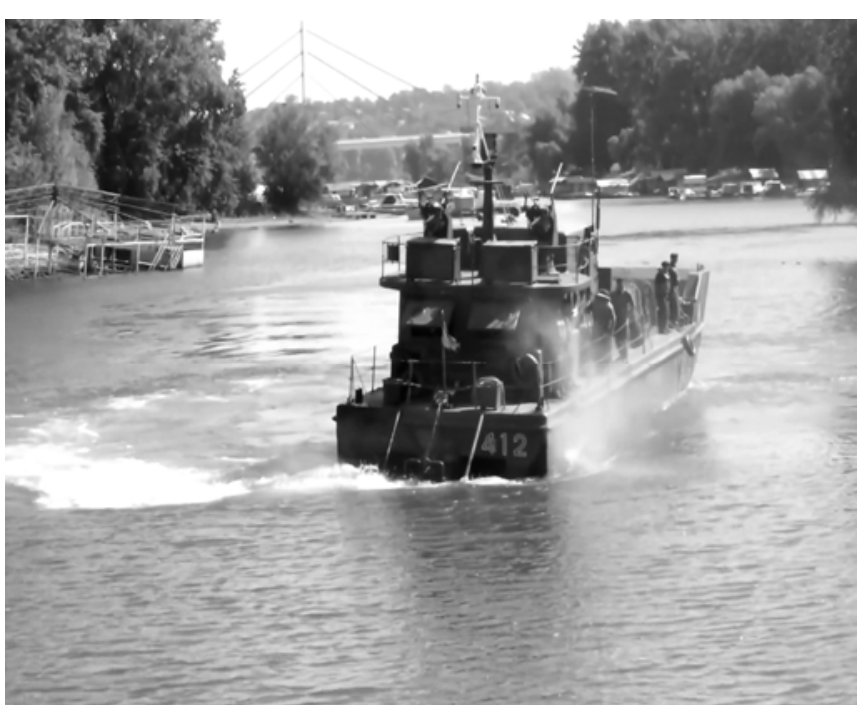

(b)

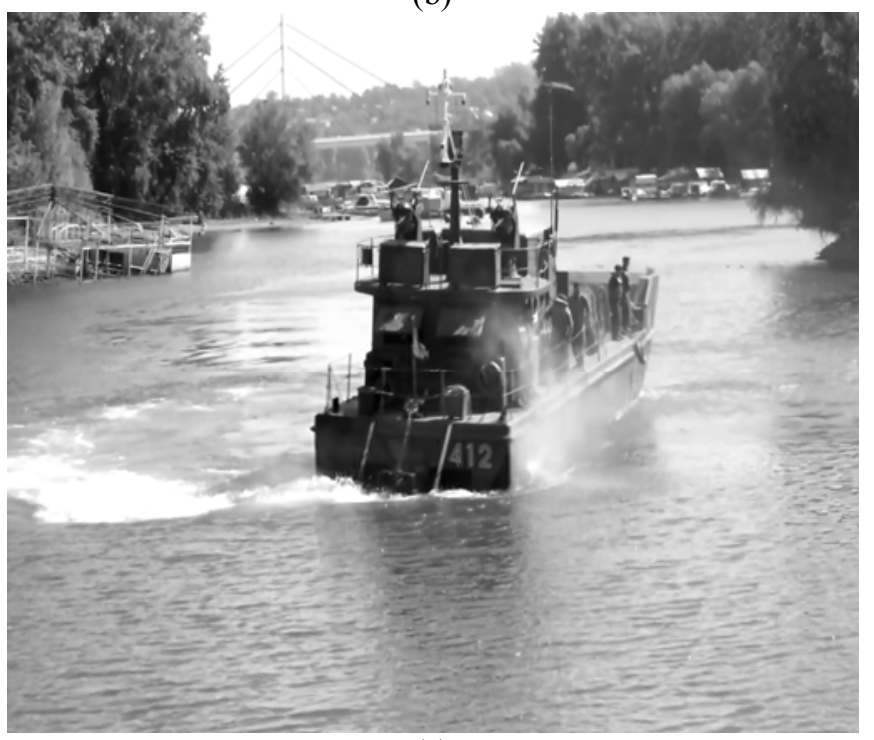

(c)

Figure 8: For HD1080p resolution video; (a) Original frame No. \#58, (b) Reconstructed frame No. \#58 using Conventional MCFI algorithm, (c) Reconstructed frame No. \#58 using Cubic MCFI algorithm.

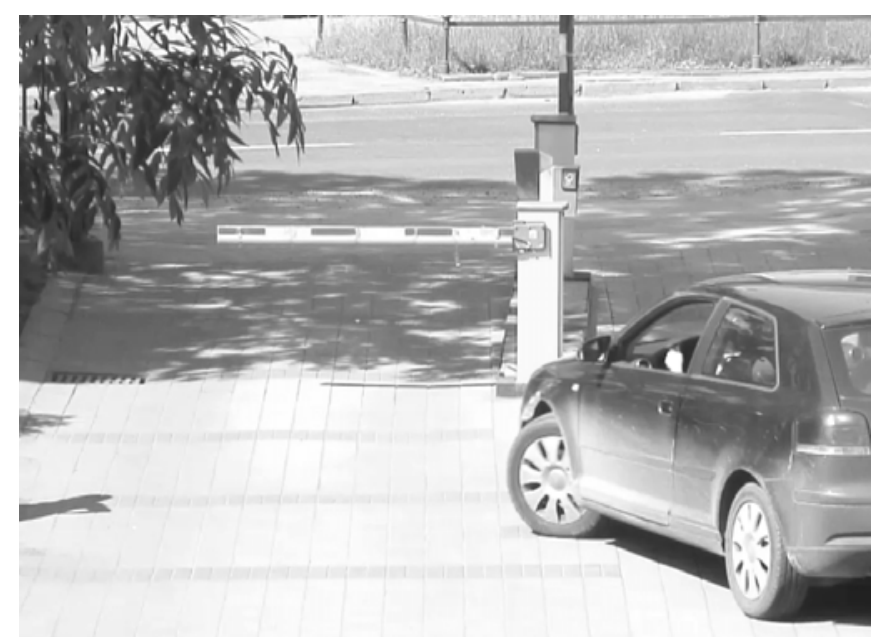

(a)

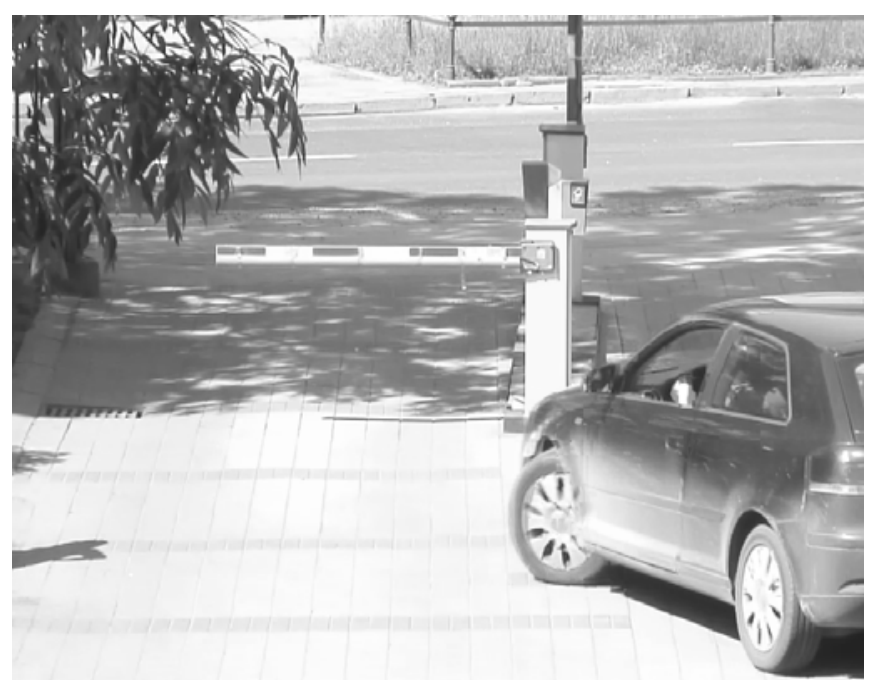

(b)

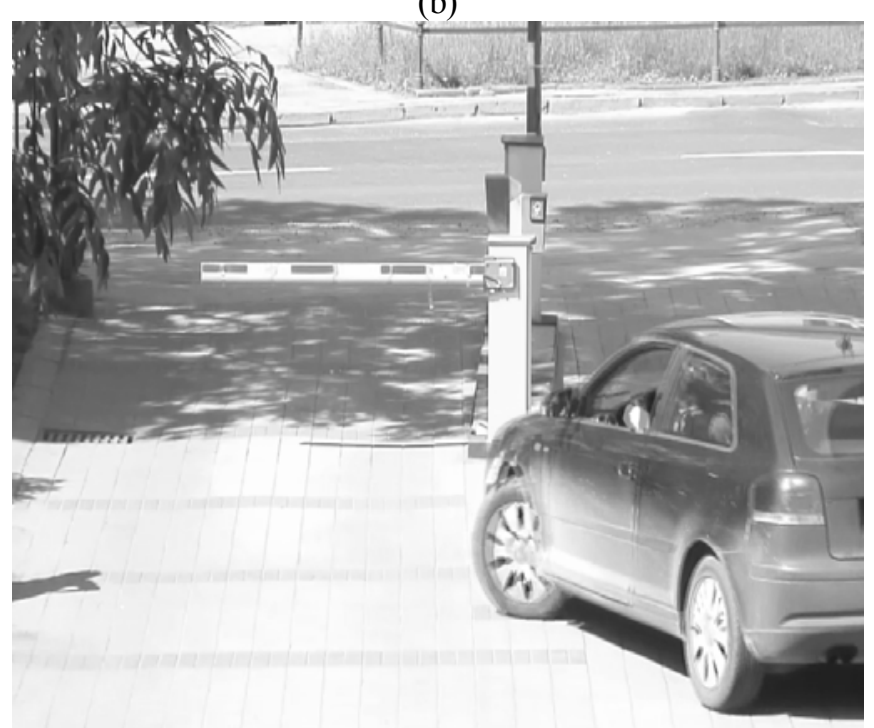

(c)

Figure 9: For HD720p resolution video; (a) Original frame No. \#32, (b) Reconstructed frame No. \#32 using Conventional MCFI algorithm, (c) Reconstructed frame No. \#32 using Cubic MCFI algorithm.

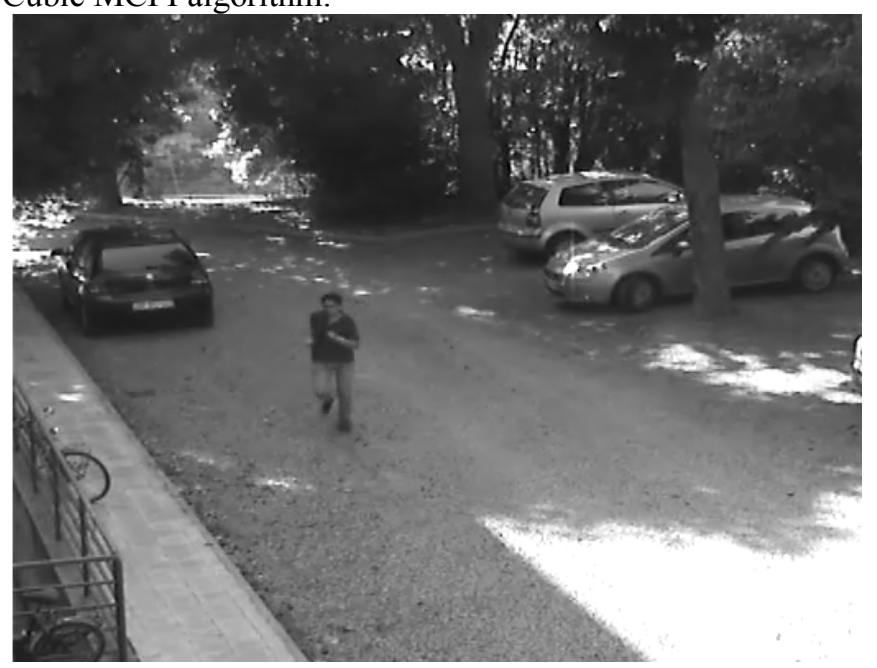

(a) 


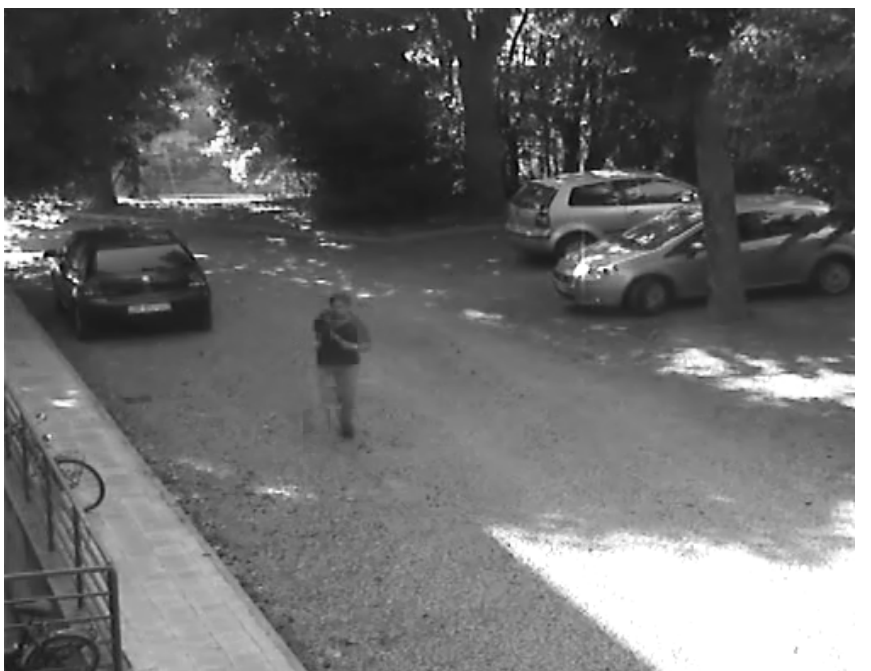

(b)

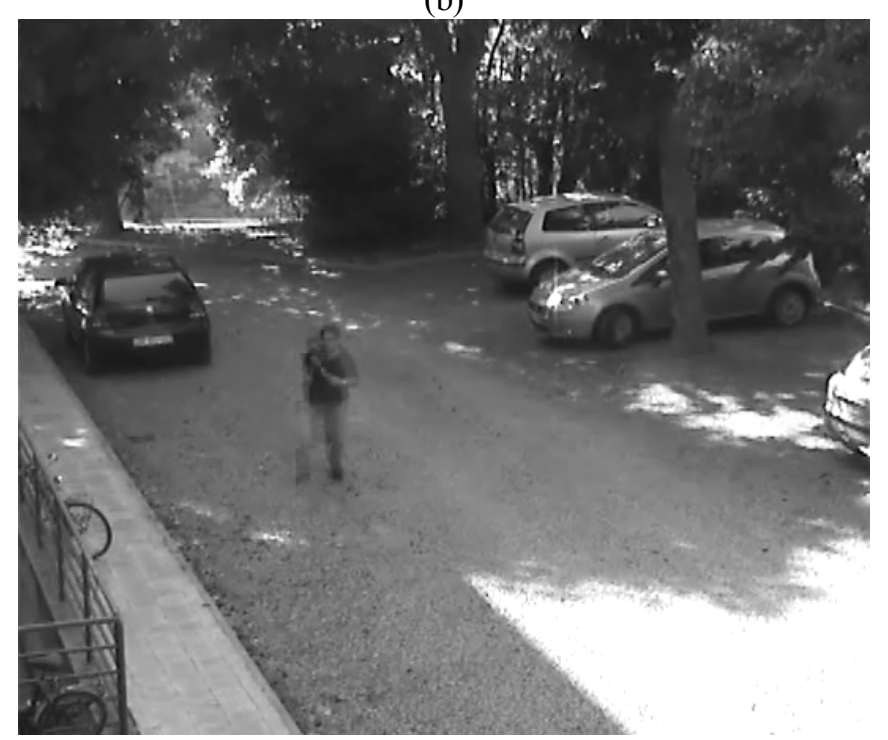

(c)

Figure 10: For VGA $640 * 480$ resolution video; (a) Original frame No. \#38, (b) Reconstructed frame No. \#38 using Conventional MCFI algorithm, (c) Reconstructed frame No. \#38 using Cubic MCFI algorithm.

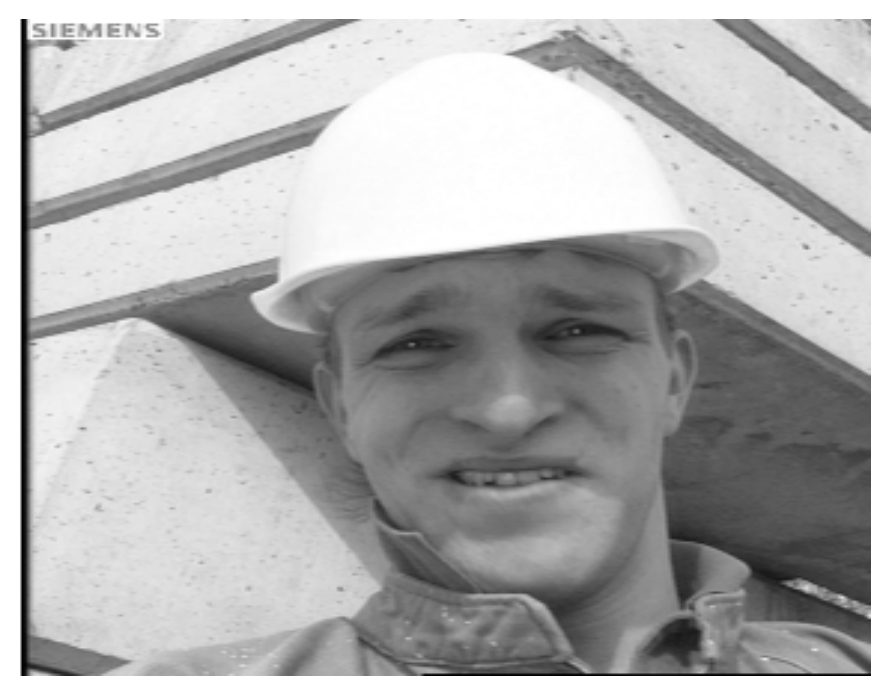

(a)

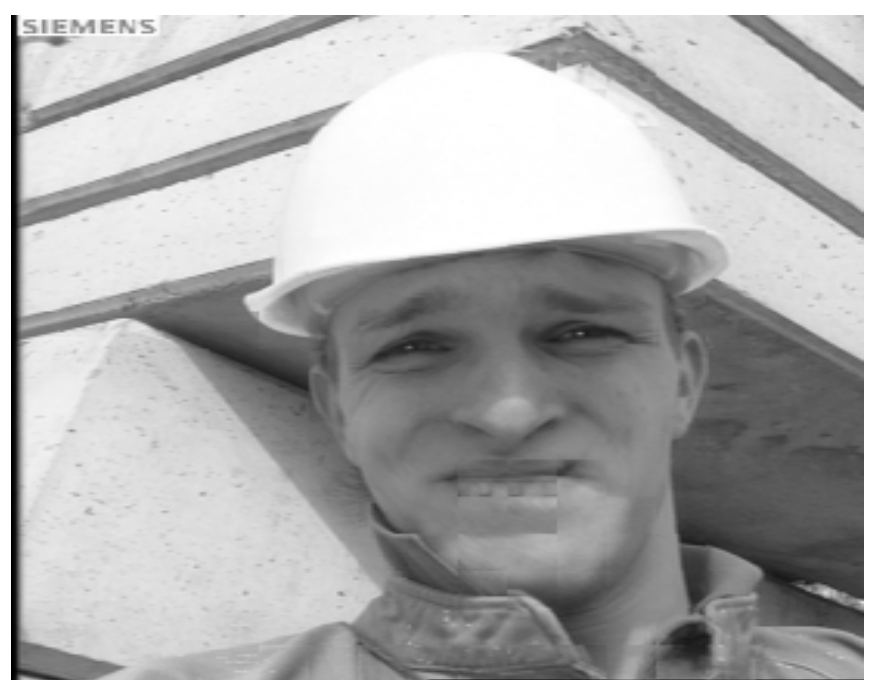

(b)

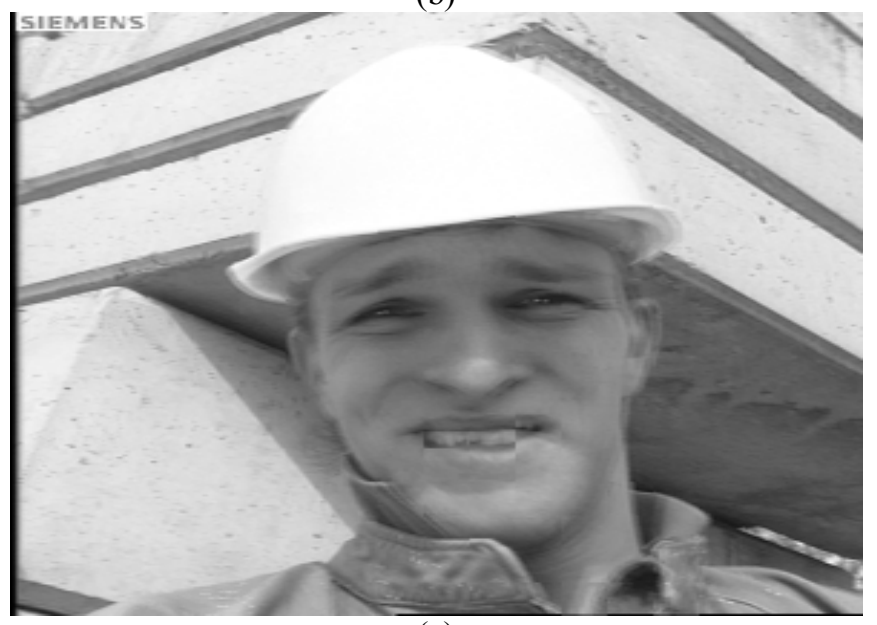

(c)

Figure 11: For HD1080p resolution video; (a) Original frame No. \#42, (b) Reconstructed frame No. \#42 using Conventional MCFI algorithm, (c) Reconstructed frame No. \#42 using Cubic MCFI algorithm.

\section{CONCLUSION}

This paper proposes a new cubic MCFI method for different video sequences with different frame sizes varies from CIF to HD 1080. Experimental results show that cubic MCFI algorithm achieved better performance than conventional algorithm qualitatively. From Table II and Figure 2-Figure 3, it can be concluded that proposed algorithm improves PSNR by approximate $3 \mathrm{~dB}$ and SSIM by $5 \%$ than conventional algorithm. Results can be further improved by using recent motion estimation methods like hierarchical search method or 3D recursive search method or combination of both methods in Motion Estimation block of temporal frame interpolation process.

\section{REFERENCES}

[1] Kang, Suk-Ju, Kyoung-Rok Cho and Young Hwan Kim, 2007, "Motion compensated frame rate up- 
conversion using extended bilateral motion estimation." Consumer Electronics, IEEE Transactions on 53.4: pp.1759-1767.

[2] Wang, Jinsong, Nilesh Patel and William Grosky, 2004, "A fast block-based motion compensation video frame interpolation." Signals, Systems and Computers, 2004, Conference Record of the ThirtyEighth Asilomar Conference on. Vol. 2. IEEE.

[3] Choi, Byung-Tae, Sung-Hee Lee and Sung-Jea Ko., 2000, "New frame rate up-conversion using bidirectional motion estimation." Consumer Electronics, IEEE Transactions on 46.3: 603-609.

[4] Sung-Hee Lee, Yoon-Cheol Shin, Seungjoon Yang, Heon-Hee Moon and Rae Hong Park, 2002, "Adaptive motion-compensated interpolation for frame rate up-conversion," Consumer Electronics, IEEE Transactions on (Volume :48, Issue: 3), Pages 444-450.

[5] Jeong, Seong-Gyun, Chul Lee and Chang-Su Kim, 2013, "Motion-compensated frame interpolation based on multihypothesis motion estimation and texture optimization." Image Processing, IEEE Transactions on 22.11: 4497-4509.

[6] Yang, Xiaohui, et al, 2014, "Depth-Assisted Frame Rate Up-Conversion for Stereoscopic Video." Signal Processing Letters, IEEE 21.4: 423-427.

[7] Han, Rui, and Aidong Men, 2013, "Frame rate upconversion for high-definition video applications." Consumer Electronics, IEEE Transactions on 59.1: 229-236.

[8] Dikbas, Salih and Yucel Altunbasak, 2013, "Novel true-motion estimation algorithm and its application to motion-compensated temporal frame interpolation." Image Processing, IEEE Transactions on 22.8: 2931-2945.

[9] Huang, Ai-Mei and Truong Nguyen, 2009, "Motion vector processing using the color information." Image Processing (ICIP), 2009 16th IEEE International Conference on. IEEE.

[10] Anton Veselov, Marat Gilmutdinov, 2014, "Iterative Hierarchical true motion Estimation For temporal Frame Interpolation," Multimedia Signal Processing (MMSP), 2014 IEEE $16^{\text {th }}$ International Workshop on, 22-24 Sept., pp. 1-6.

[11] Dong Yoon Kim and Hyun Wook Park, 2015, "An Efficient Motion-Compensated frame Interpolation method using Temporal Information for Highresolution Videos," Display Technology, Journal of (Volume: 11, Issue: 7), pp. 580-588.

[12] Akin A.; Cetn M.; Ozcan Z.; Erbagci B.; 2012, “An Adaptive bilateral motion Estimation algorithm and its hardware architecture," Consumer Electronics, IEEE Transactions, Volume: 58, Issue: 2, Pages: $712-720$.

[13] Yong Guo, Zhiyong Gao, Li Chen and Xiaoyun Zhang, 2011, "Occlusion Handling Frame Rate Upconversion," Image and Signal Processing and Analysis (ISPA), $7^{\text {th }}$ International Symposium on, 46 Sept., Pages 149-152.
[14] Keys R., 1981, "Cubic convolution interpolation for digital image processing," Acoustic, Speech and Signal Processing, IEEE transaction on, (Volume: 29, Issue: 6), pp. 1153-1160.

[15] Z. Wang, H. R. Sheikh, and A. C. Bovik, 2003, "Objective video quality assessment," in Handbook of Video Database; Design and Application CRC Press.

[16] Jain, Jaswant $\mathrm{R}$ and Anil K. Jain, 1981, "Displacement measurement and its application in interframe image coding." Communications, IEEE Transactions on 29.12: 1799-1808.

[17] Richardson, Iain E. H. 264 and MPEG-4 video compression, 2004, "video coding for nextgeneration multimedia", John Wiley \& Sons.

[18] Rafael C. González, Richard Eugene Woods, 2002 "Digital image processing", Prentice Hall, Edition 2.

[19] Naveen, C.; Gupta, T. V. S.; Satpute, V. R.; Gandhi, A. S., "A simple and efficient approach for medical image security using chaos on EZW", Advances in Pattern Recognition (ICAPR), 2015 Eighth International Conference on, 4-7. 2015, IEEE, pp. 16.

[20] Ch. Naveen, V. R. Satpute, A. G. Keskar, " An Efficient Low Dynamic Range Image Compression using Improved Block Based EZW", IEEE Workshop on Computational Intelligence, IEEEWCI 2015, 14-17 December 2015, IIT Kanpur, India., in press.

[21] For Video database (Videos Downloaded from database) Link: http://see.xidian.edu.cn/vipsl/database_Video.html

[22] For Video database (Videos Downloaded from database) Link: http://www.openvisor.org/

[23] For Video database (Videos Downloaded from database) Link: http://vq.kt.agh.edu.pl/videoLibrary.html

[24] For Video database (Videos Downloaded from database) Link: www.all-free-download.com 